\title{
Near-Perfect Absorbing Copper Metamaterial for Solar Fuel Generation
}

\author{
Authors: Joel Y.Y. Loh ${ }^{1, \dagger}$, Mahdi Safari ${ }^{1, \dagger}$, Chengliang Mao², Camilo J. Viasus ${ }^{2}$, George V. \\ Eleftheriades ${ }^{1}$, Geoffrey A. Ozin ${ }^{2}$, Nazir P. Kherani ${ }^{1,3 *}$. \\ Affiliations: \\ 1Department of Electrical and Computing Engineering, University of Toronto, Toronto, Ontario M5S 3G4, \\ Canada. \\ 2Department of Chemistry, 80 Saint George Street, University of Toronto, Toronto, M5S 3H6, Ontario, \\ Canada. \\ ${ }^{3}$ Department of Material Science and Engineering, University of Toronto, Toronto, Ontario M5S 3E4, \\ Canada. \\ ${ }^{*}$ Correspondence to: nazir.kherani@utoronto.ca \\ $\dagger$ J.YY. Loh and M. Safari contributed equally to the manuscript.
}

\section{Supplementary Information}

\section{Materials and methods}

\section{Reactor Sequence}

In the batch reactor configuration, the reactor was initially vacuum pumped to $2.0-2.2 \times 10^{-2}$ mbar, before heating the reactor to the desired temperature. A K-type thermocouple probe was affixed within the reactor and the tip was in contact with the corner of the sample substrate. Subsequently, $\mathrm{H}_{2}$ and $\mathrm{CO}_{2}$ were introduced at pressure ratios of 4.2 to 1 . The reaction time under dark or illumination is typically $10-20$ hours before injecting the sample atmosphere into the GC (SRI 8610 Gas Chromatograph system). A standard calibrant cylinder with Methanol (523 ppm), $\mathrm{CO}_{2}$ (520 ppm), $\mathrm{CO}$ (524 ppm) and $\mathrm{CH}_{4}$ (502 ppm) was used to quantify the reaction products.

In order to confirm if the methanol product was generated from the $\mathrm{CO}_{2}$ precursor, ${ }^{13} \mathrm{C}$ isotope labelled $\mathrm{CO}_{2}$ was used. After a reactor temperature of $230^{\circ} \mathrm{C}$ over 14 hours, a sample gas was analyzed with a GC-Mass-Spectrometer, and the acquired mass spectrum was compared to a database calibrated mass spectrum according to the fragmentation of a ${ }^{13} \mathrm{CH}_{3} \mathrm{OH}$ molecule.

\section{Gas Chromatograph-Mass Spectroscopy Isotope Testing}

Isotopically labelled experiments were performed to determine the origin of $\mathrm{CH}_{3} \mathrm{OH}$ and $\mathrm{CO}$ and rule out any possible artifact in the process. The gas samples were analyzed using a GCMS spectrometer (Agilent 7890B-5977A MSD, using He as carrier gas) equipped with an automated injection gas sampling valve over three capillary columns (Molsieve, HP-PLOT/Q+PT and DB- 
FFAP) to improve separation and quantification. The signal peaks at a retention time (rt) of 3.49 and 10.10 minutes confirmed the presence of ${ }^{13} \mathrm{CO}$ and ${ }^{13} \mathrm{CH}_{3} \mathrm{OH}$, respectively, generated from ${ }^{13} \mathrm{CO}_{2}$ and $\mathrm{H}_{2}$ over the $\mathrm{ZnO} / \mathrm{Cu}$ metasurface. Standard ${ }^{12} \mathrm{CH}_{3} \mathrm{OH}$ and ${ }^{13} \mathrm{CH}_{3} \mathrm{OH}$ samples from Sigma-Aldrich were used as control experiments.

The expected $\mathrm{CH}_{3} \mathrm{OH}$ fragmentation mass spectrum and normalized intensities for all analyzed samples are presented in Table S1 and Fig. S1A. The experimental isotopic fragmentations for ${ }^{13} \mathrm{CH}_{3} \mathrm{OH}$ are shown in Fig. S1C. The +1 mass increment on the isotopic experiment and the signal intensities confirmed that methanol's presence came from the ${ }^{13} \mathrm{CO}_{2}$ used. This behavior is characteristic of ${ }^{13} \mathrm{CH}_{3} \mathrm{OH}$ fragmentation (Fig. S1B-C), thus undoubtedly positively ruling out any adventitious carbon source. 
Table S1. Expected fragmentation of ${ }^{12} \mathrm{CH}_{3} \mathrm{OH}$ of standard, ${ }^{13} \mathrm{CH}_{3} \mathrm{OH}$ of standard and metasurface.

\begin{tabular}{|c|c|c|c|c|c|c|}
\hline $\mathrm{m} / \mathrm{z}(+)$ & 16 & 29 & 30 & 31 & 32 & 33 \\
\hline Ion species & ${ }^{13} \mathrm{CH}_{3}{ }^{+}$ & ${ }^{13} \mathrm{CO}^{+}$ & ${ }^{13} \mathrm{CHO}^{+}$ & ${ }^{13} \mathrm{CHOH}^{+}$ & ${ }^{13} \mathrm{CH}_{2} \mathrm{OH}^{+}$ & ${ }^{13} \mathrm{CH}_{3} \mathrm{OH}^{+}$ \\
\hline${ }^{12} \mathrm{CH}_{3} \mathrm{OH}$ Control (\%) & 0.52 & 49 & 7.0 & 100 & 78.5 & 1.7 \\
\hline${ }^{13} \mathrm{CH}_{3}$ OH Sigma (\%) & 9.7 & 4.8 & 52.8 & 7.6 & 100 & 71.0 \\
\hline${ }^{13} \mathrm{CH}_{3} \mathrm{OH}$ Metasurface (\%) & 9.3 & 6.1 & 52.6 & 8.8 & 100 & 68.2 \\
\hline
\end{tabular}

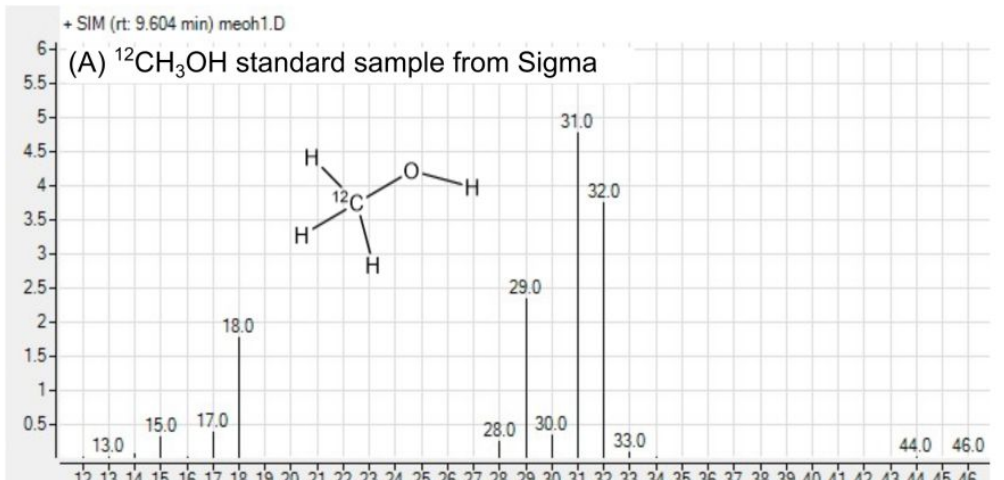

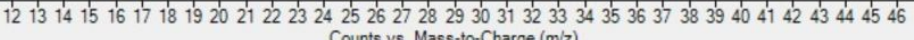
Counts vs. Mass-to-Charge $(\mathrm{m} / \mathrm{z})$
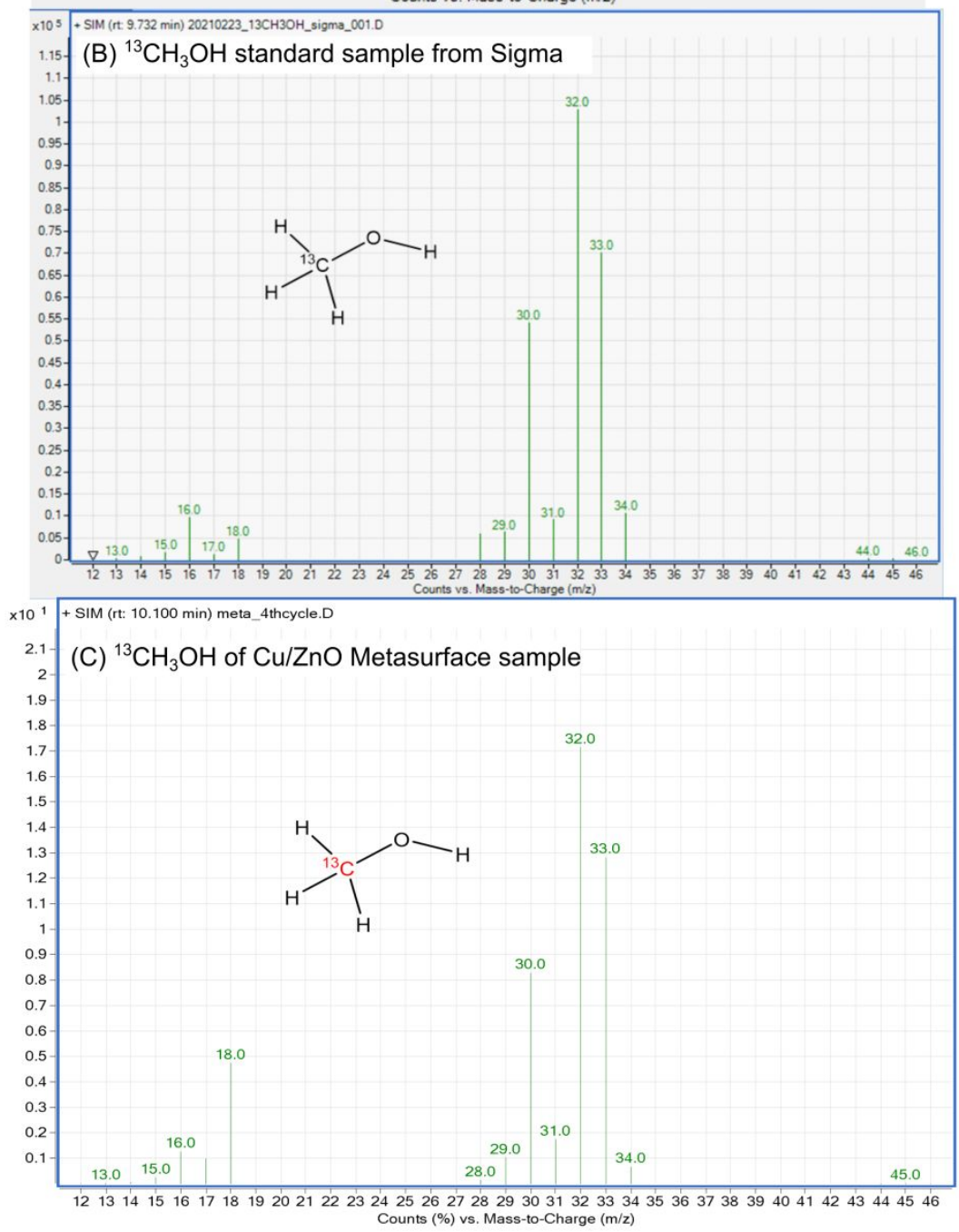

Fig. S1.

(A, B) Reported ${ }^{12} \mathrm{CH}_{3} \mathrm{OH}$ and ${ }^{13} \mathrm{CH}_{3} \mathrm{OH}$ fragmented species and the associated $\mathrm{m} / \mathrm{z}$ from the measured calibration standard from Sigma.

(C) Mass spectrum of the signal collected at 10.10 min retention time after testing the metasurface at $230^{\circ} \mathrm{C}$ under reactant ${ }^{13} \mathrm{CO}_{2}$ and $\mathrm{H}_{2}$ atmosphere. 


\section{$\underline{H}_{2}$ baking.}

In order to remove excess carbon contamination from the electron beam lithography and evaporation process, it was necessary to bake the metamaterial sample at mild temperatures of $150-160^{\circ} \mathrm{C}$ over several days in only $\mathrm{H}_{2}$ atmosphere. The $\mathrm{H}_{2}$ pressure was $38-42 \mathrm{psi}$ and introduced after vacuum pumping down to $2.1 \times 10^{-2}$ mbar. Following the baking procedure which was carried out over 4 days, the sample was cooled naturally in vacuum, and subsequent $\mathrm{CO}_{2}$ reduction measurements were carried out.

\section{Arrhenius Plots}

(A)

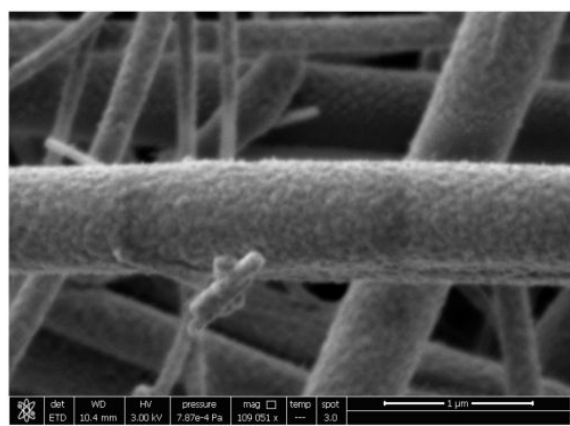

(C)

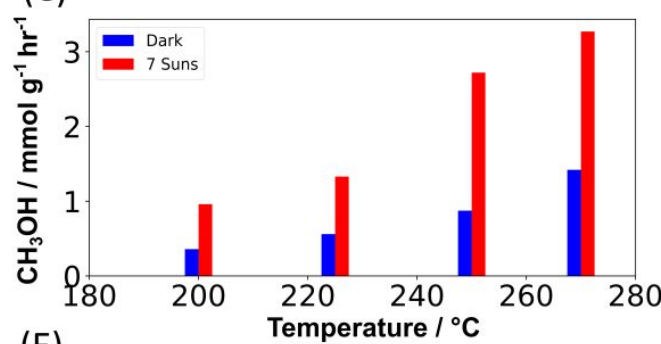

$(\mathrm{E})$
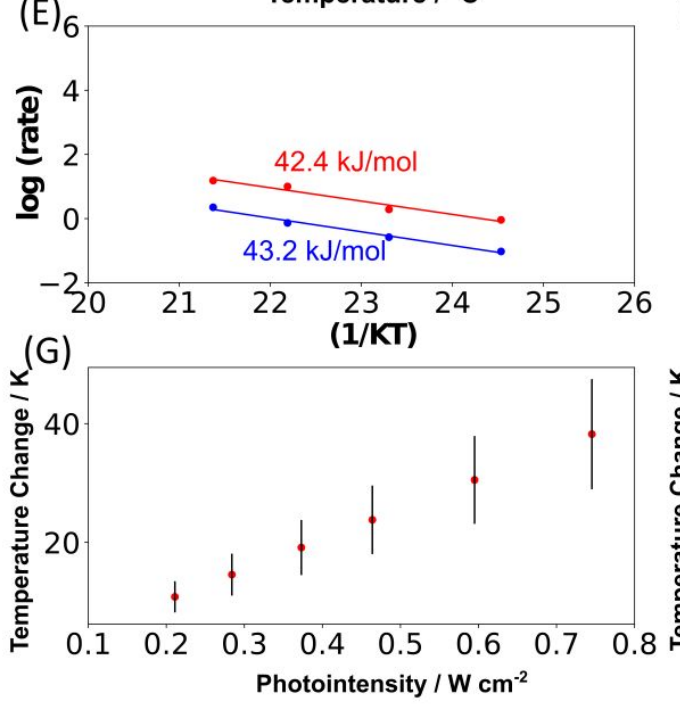

(B)

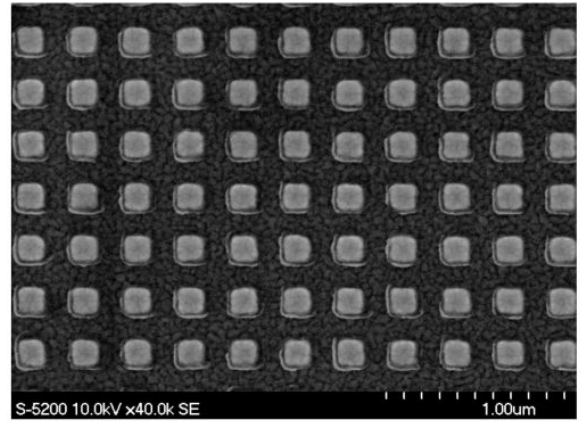

(D)
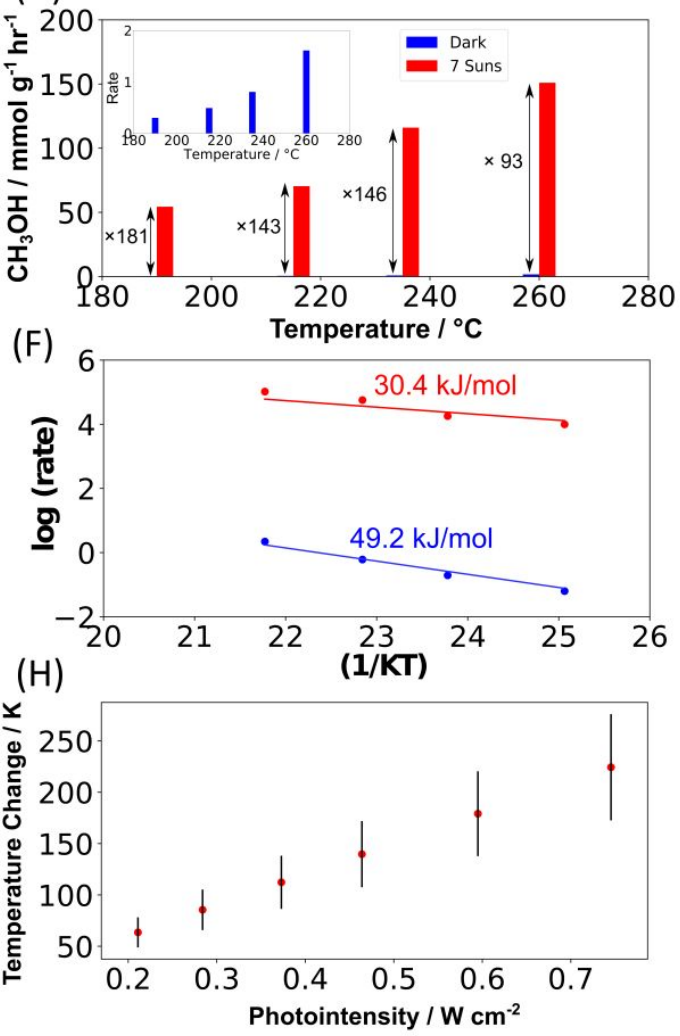

Fig. S2. (A) and (B) are additional SEM images of the $\mathrm{ZnO} / \mathrm{Cu}$ layers deposited on quartz fiber substrate (Whatman 1823-010 Glass Microfiber Binder Free Filter) and as synthesized into a cubical metasurface 
structure. (C) and (D) are the methanol production rate of the $\mathrm{ZnO} / \mathrm{Cu}$ film on quartz fiber surface and metasurface under dark and under 7.1 suns photo-illumination, respectively. (E, F) The calculated thermal associated activation energies for the film on quartz fiber surface and metasurface under dark ranges are $43.2 \mathrm{~kJ} / \mathrm{mol}$ and $49.2 \mathrm{~kJ} / \mathrm{mol}$, which are fairly similar. $(\mathbf{G}, \mathbf{H})$ Calculated temperature rise based on modified Arrhenius equation with photo-intensities converted to an equivalent temperature rise, for thin film on fiber and metasurface respectively.

The mass of the nano-cubes over a $1 \mathrm{~cm}$ by $1 \mathrm{~cm}$ area as fabricated by electron beam lithography, was $(2.5 \pm 0.21) \times 10^{-5} \mathrm{~g}$ and the mass of the film deposited by electron beam evaporation over a $2 \mathrm{~cm}$ by $2 \mathrm{~cm}$ fiber area, as measured by a microbalance, was $4.7 \times 10^{-4} \mathrm{~g}$. The surface area of the nano-cube array $\left(39799 \mathrm{~cm}^{2} / \mathrm{g}\right)$ is thus determined to be greater than that of the granular film on quartz fiber $\left(9918 \mathrm{~cm}^{2} / \mathrm{g}\right)$ by a factor of 4 . Since the methanol rate of the metasurface under dark is comparable to the granular film on quartz fiber under dark by a factor of $0.7-1.5$, it can be assumed that the higher activity can be attributed to the increased surface area of the structured over the unstructured material. It is to be noted that the aim of this investigation is to determine the efficacy of a metamaterial structure interacting with electromagnetic radiation, compared to dark. Hence, the photo-induced enhancement in rate is the primary axis of interest. According to various illumination studies on copper/zinc oxide or copper/zinc oxide/aluminum oxide (CZA) catalysts, the photo-induced enhancement factors were reported to be in the range of $\sim 1.45$ and $\sim 1.6$ under 600 and $580 \mathrm{~mW} / \mathrm{cm}^{2}$ photointensities ${ }^{23,24}$, respectively. These factors are of the same order of magnitude of 2,3-3.1 between light and dark of the granular film on fiber surface as shown here. Hence, underwhelming photo enhancement factors are commonly seen in the literature.

When the illuminated and dark associated rates of the metasurface are compared, it can be seen that the photo associated enhancement is greater at lower reactor temperatures. This may be linked to the high activation temperature of copper/zinc oxide catalysts for methanol synthesis ${ }^{37}$, which typically initializes from at least $225-240^{\circ} \mathrm{C}$, given that formate hydrogenation presents a significant barrier ${ }^{3839}$. Hence a small illumination induced temperature increase from just below the threshold temperature presents a substantially greater product rate. (F) show that the Arrhenius activation energy of the $\mathrm{ZnO} / \mathrm{Cu}$ metasurface is significantly reduced to $30.4 \mathrm{~kJ} / \mathrm{mol}$. To determine the effective local temperature increase experienced by the $\mathrm{ZnO} / \mathrm{Cu}$ nano-cubes $(\mathbf{G})$ as a result of the absorption enhanced plasmonic effect as discussed in the main text, the modified Arrhenius equation under a range of photo-intensities and temperatures, showed relatively consistent temperature rises at low photo-intensities but diverges at higher photo intensities, which may be due to increased joule heating or due to high indirect charge 
transfer rates to an increased electric field intensity associated with plasmonic-absorption coupling. The granular film on quartz fiber showed a wider scatter in the calculated temperature increase values $(\mathbf{H})$, possibly due to the overall low temperature increase and the sensitivity of the exponent function. High temperatures have been reported for various metamaterial absorbers such as $\mathrm{Au} / \mathrm{Ge}_{2} \mathrm{Sb}_{2} \mathrm{Te}_{5}$ with $160^{\circ} \mathrm{C}$ under 9 suns ${ }^{16}$, and $\mathrm{Ti} / \mathrm{MgF}_{2} / \mathrm{W}$ with $400^{\circ} \mathrm{C}$ under 25 suns ${ }^{40}$. However, the optical absorption of $\mathrm{Ti}_{/} / \mathrm{MgF}_{2} / \mathrm{W}$ absorber is invariant only from 0 to $30^{\circ}$ incident angle, whereas the $\mathrm{Au} / \mathrm{Ge}_{2} \mathrm{Sb}_{2} \mathrm{Te}_{5}$ absorber shows absorption angular invariance extending to $80^{\circ}$. Hence, our metasurface likely shows good temperature conversion factors, comparable to other metamaterial absorbers.

\section{X-ray Photoelectron Spectroscopy}

The XPS spectra of the metamaterial structures are shown in Fig. S3. The Cu $2 p$ spectrum (A) shows little to no satellite peaks, the peaks of $932.2 \mathrm{eV}$ and $952.0 \mathrm{eV}$ are not in line with the values of $933.7 \mathrm{eV}$ and $953.6 \mathrm{geV}$ associated with $\mathrm{Cu}^{2+} 2 \mathrm{p}_{3 / 2,1 / 2}$ core levels ${ }^{41}$. Additionally, the Cu LMM-2 peak (B) is located at $567.5 \mathrm{eV}$, which is associated with $\mathrm{Cu}^{0}$ metal, and shows no well-defined peaks located at $570 \mathrm{eV}$ (associated with $\mathrm{Cu}^{+}$of $\left.\mathrm{Cu}(\mathrm{I})_{2} \mathrm{O}\right)^{42}$. It is thus likely that the copper cubes do not experience significant ambient oxidation after fabrication. The Zn2p and O1s spectra (C, D) shows the presence of $\mathrm{Zn}(\mathrm{OH})_{2}$ and $\mathrm{ZnO}$ where the $\mathrm{Zn} 2 \mathrm{p}$ peaks are close to the reported values of for $\mathrm{Zn}(\mathrm{OH})_{2}$ at $1021.8 \mathrm{eV}$ while the $01 \mathrm{~s}$ spectrum consist of $\mathrm{Zn}-\mathrm{O}$ lattice bonds at $530.04 \mathrm{eV}$ and $\mathrm{Zn}-\mathrm{OH}$ species at $531.4 \mathrm{eV}^{43,44}$. The $\mathrm{C} 1 \mathrm{~s}$ spectrum (F) showed mostly a $\mathrm{C}-\mathrm{C}$ species associated with adventitious carbon. 

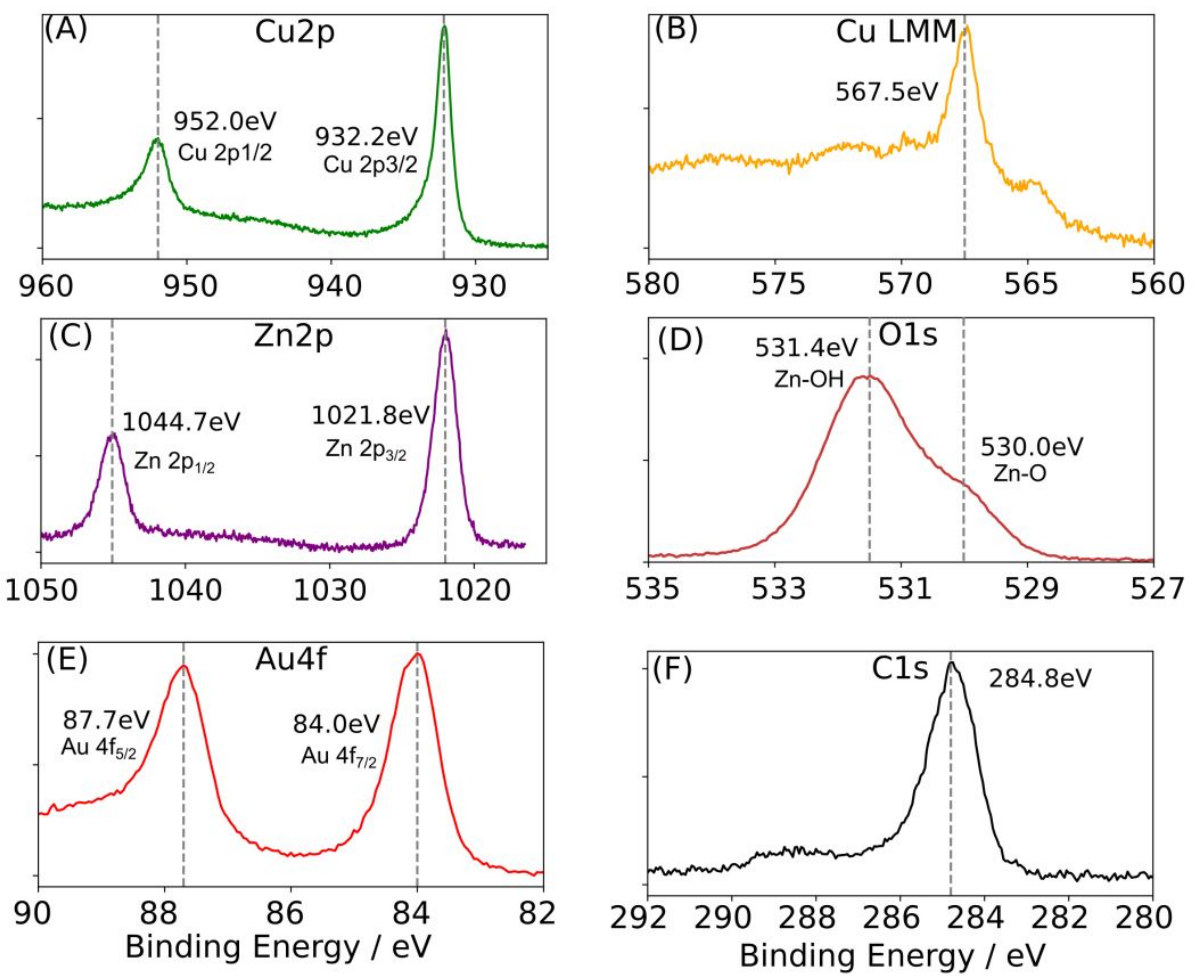

Fig. S3. XPS spectra. (A,B) are Copper Cu2p and LMM-2 Auger transition spectra. (C) is Zinc $\mathrm{Zn} 2 \mathrm{p}$ and $(\mathbf{D})$ is $\mathrm{O} 1 \mathrm{~s}$ spectra. (E) is Gold Au $4 \mathrm{f}$ spectrum. $(\mathbf{F})$ is Carbon C1s spectrum.

\section{FTIR Details}

Diffuse reflectance infrared Fourier transform spectroscopy (DRIFTS) experiments were performed on a Thermo Scientific Nicolet iS50 FTIR Spectrometer with a liquid nitrogen cooled $\mathrm{HgCdTe}$ (MCT) detector. 32 spectra were co-added with a resolution of $4 \mathrm{~cm}^{-1}$. The metasurface sample or granular film on quartz fiber surface sample were placed on top of the sample holder in a Harrick high-temperature reaction chamber which was equipped with ZnSe window. Light was introduced through the front viewing window of the reaction chamber. The temperature of the chamber was controlled by a Harrick ATC-024-3 Temperature Controller and a cooling water system. To compensate for oversaturation of signal from the meatasurface the detector gain was reduced. Spectra noise was minimized by Savitzky-Golay filter processing. Peak assignments were extracted from ${ }^{25}$ and ${ }^{45}$.

Table S2

\begin{tabular}{|l|l|}
\hline Wavenumber $/ \mathrm{cm}^{-1}$ & Assignment \\
\hline
\end{tabular}




\begin{tabular}{|l|l|}
\hline 1080 & ${ }^{*} \mathrm{CH}_{3} \mathrm{O}$ \\
\hline 1122 (after light) & $\mathrm{CH}_{3} \mathrm{OH}$ (Gas) \\
\hline 1168 (after light) & $\mathrm{CH}_{3} \mathrm{OH}$ (Gas) \\
\hline 1260 & $\mathrm{CO}_{3}^{2-}$ on $\mathrm{Cu}$ \\
\hline 1517 & $\mathrm{HCO}_{2}-$ on $\mathrm{Cu}$ \\
\hline 1610 & $\begin{array}{l}\mathrm{HCO}_{3} \text { on } \mathrm{ZnO} / \\
\mathrm{HCO}_{2}-\text { on } \mathrm{Cu}\end{array}$ \\
\hline 2063 & $\mathrm{CO}$ on $\mathrm{Cu}(111)$ \\
\hline 2078 & $\mathrm{CO}$ on $\mathrm{Cu}(110)$ \\
\hline 2091 & $\mathrm{CO}$ on $\mathrm{Cu}(755)$ \\
\hline
\end{tabular}

\section{$\underline{\text { X-ray Diffraction }}$}




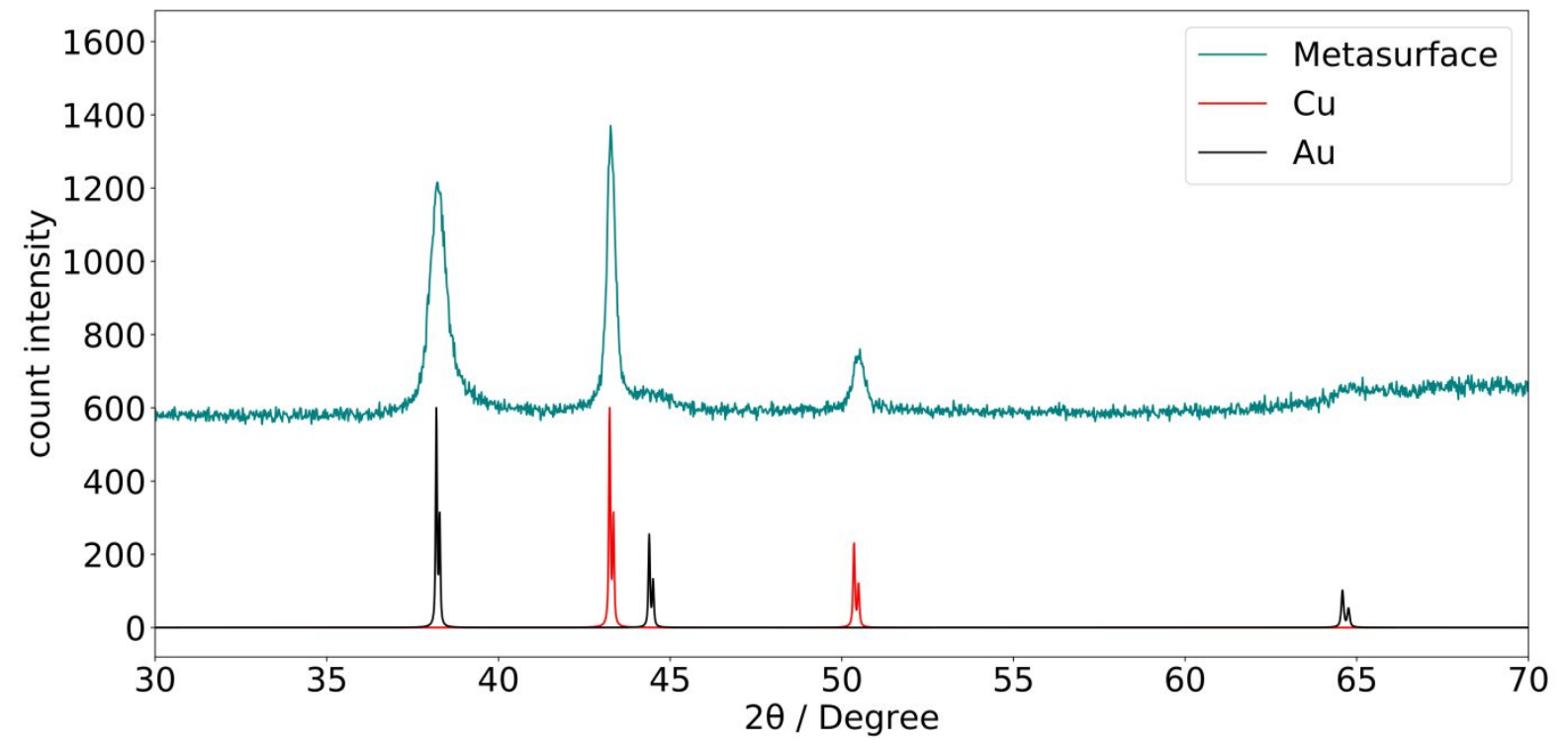

Fig. S4. XRD spectrum of the metasurface. The amount of $\mathrm{ZnO}$ material was too low to be detected. $\mathrm{Cu}$ (CIF ID mp-30) and Au (CIF ID 9008463) diffraction spectra generated by VESTA are shown in comparison.

\section{Metasurface Design and Characterization.}

(A)

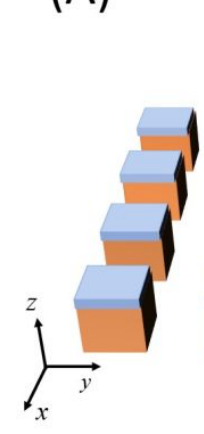

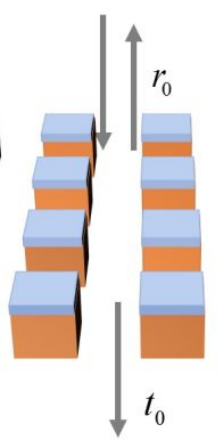

(B)

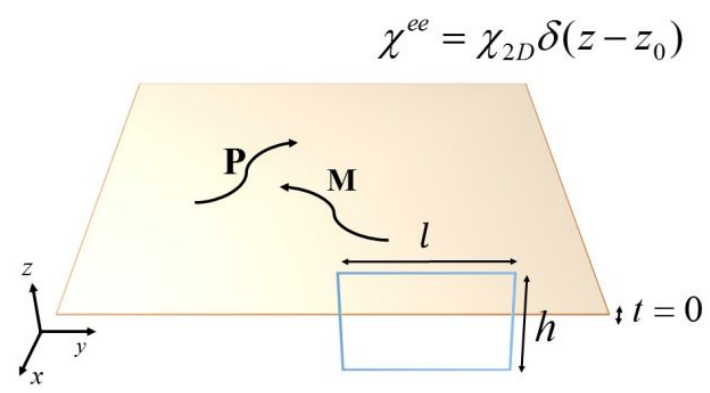

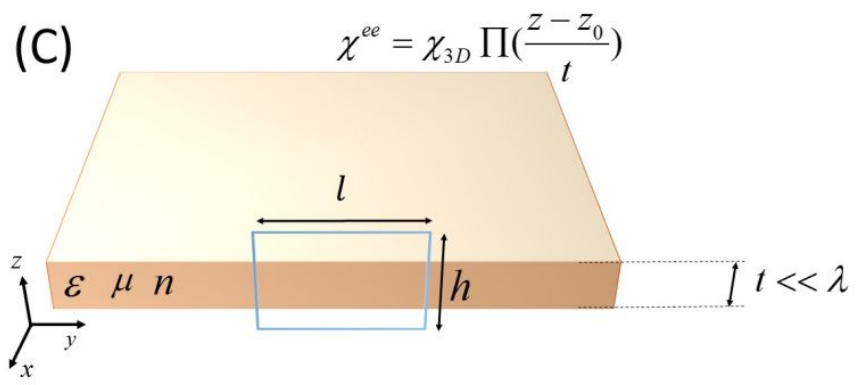

Fig. S5 Schematic view of (A) nano-cube layer standing in free space, (B) generalized sheet boundary condition with the induced electric $(\mathrm{P})$ and magnetic $(\mathrm{M})$ polarizations, and $(\mathbf{C})$ homogenized $3 \mathrm{D}$ effective layer. 
The proposed near-perfect metasurface absorber is composed of multilayer stack of catalyst and optical materials, wherein the top layer consists of nano-cubes comprising $\mathrm{ZnO}$ and $\mathrm{Cu}$ while the back mirror consists of uniform layers of $\mathrm{Au}$ and $\mathrm{Cu}$. Here, we use the generalized sheet boundary condition as the primary mean to extract the homogenized effective optical properties of the top $\mathrm{ZnO} / \mathrm{Cu}$ nano-cube layer standing in free space without the back mirror (Fig. S5). Based on the generalized sheet boundary condition, we first assume that the nano-cube metasurface layer thickness is thin when compared to the operating wavelength. As a result, electromagnetic fields and polarizations can be homogenized over the thickness. As an example, we make reference to the characterization of plasmonic gold nanospheres as explained in detail using the mentioned approach ${ }^{32}$. Using this method, we derive the surface susceptibility tensors induced over the nano-cube layer using the reflection and transmission coefficients extracted from numerical simulations.

$$
\begin{aligned}
& \chi_{x x}^{e e}=\frac{1}{\eta_{0}} \frac{-2 i}{\omega} \frac{r_{0}+t_{0}-1}{r_{0}+t_{0}+1} \\
& \chi_{y y}^{m m}=\eta_{0} \frac{-2 i}{\omega} \frac{r_{0}-t_{0}+1}{r_{0}-t_{0}-1} \\
& \sin ^{2} \theta \eta_{0} \chi_{z z}^{e e}=\frac{\chi_{y y}^{m m}}{\eta_{0}}-\frac{-2 i}{\omega} \frac{r_{\theta}-t_{\theta}+1}{r_{\theta}-t_{\theta}-1}
\end{aligned}
$$

where $r_{0}$ and $t_{0}$ are the reflection and transmission coefficients at normal incidence and subscript $\theta$ represents the incident angle. $\chi_{z z}^{e e}, \chi_{y y}^{m m}, \chi_{x x}^{e e}$ are the diagonal elements of the surface susceptibility tensors $\overline{\bar{\chi}}^{e e}$ and $\overline{\bar{\chi}}^{m m}$, which define the induced electric and magnetic polarizations through the following equations

$$
\begin{aligned}
& \mathbf{P}=\overline{\bar{\chi}}^{e e} \mathbf{E}^{\mathbf{a v}}+\overline{\bar{\chi}}^{e m} \mathbf{H}^{\mathrm{av}} \\
& \mathbf{M}=\overline{\bar{\chi}}^{m e} \mathbf{E}^{\mathrm{av}}+\overline{\bar{\chi}}^{m m} \mathbf{H}^{\mathrm{av}}
\end{aligned}
$$

Several physical assumptions underlie the calculations, these are:

1) Since there is no reason for a bianisotropic response, magnetoelectric and electromagnetic susceptibility tensors are assumed to be zero $\left(\overline{\bar{\chi}}^{e m}=\overline{\bar{\chi}}^{e m}=0\right)$.

2) Due to the fact that nano-cubes are symmetrical and uniformly distributed with respect to $x$ and $y$ axes, tangential susceptibility components should be equal $\chi_{x x}^{e e}=\chi_{y y}^{e e}, \chi_{x x}^{m m}=\chi_{y y}^{m m}$.

3) Finally, due to the symmetry and reciprocity of the nano-cube layer, no polarization transformation can take place and all the cross components of the susceptibility tensors vanish 
(i.e., $\quad \chi_{x y}^{e e}=\chi_{y x}^{e e}=\chi_{z y}^{e e}=\chi_{z x}^{e e}=\chi_{x z}^{e e}=\chi_{y z}^{e e}=\chi_{x y}^{m m}=\chi_{y x}^{m m}=\chi_{z y}^{m m}=\chi_{z x}^{m m}=\chi_{x z}^{m m}=\chi_{y z}^{m m}=0$ ), which can be confirmed by lack of cross components in the reflection and transmission coefficients in the numerical simulations.

4) It is worth noting that using TM polarization incident waves in the numerical simulation one can derive all the susceptibility components (i.e., $\chi_{x x}^{e e}=\chi_{y y}^{e e}, \chi_{x x}^{m m}=\chi_{y y}^{m m}, \chi_{z z}^{e e}$ ) except for the normal magnetic component (i.e., $\chi_{z z}^{m m}$ ) which can be derived under oblique TE-polarized incident waves Eq. (S3) (for more information regarding the characterization technique see ${ }^{32}$ ).

To match the refractive index of the nano-cube layer with that of the surrounding medium, we need to characterize the refractive index first. In the main manuscript we have shown that the effective medium approach and Maxwell-Garnet mixing equation can be used as a simple design tool. However, in order to better understand the effect of each layer and characterize the refractive index of the metasurface more accurately one ought to calculate the homogenized effective permittivity $(\varepsilon)$, permeability $(\mu)$, and refractive index $(n)$ of the nano-cube layer based on the induced susceptibility tensors derived from Eqs. (S1-S2). The relationships between the volumetric susceptibilities, permittivity and permeability are described in the following way: $\varepsilon_{r}=\left(1+\chi_{3 D}^{e e}\right), \mu_{r}=\left(1+\chi_{3 D}^{m m}\right)$. Previously, we assumed that the nano-cube layer is a thin surface relative to the operating wavelength and derived the induced susceptibilities Eqs. (S1-S2) from the reflective and transmission coefficients. Now, in order to take into account the thickness and calculate the homogenized properties, we need to average the derived surface susceptibilities over the thickness of the layer to obtain the volumetric or 3D susceptibilities. We can simply start from the integral form of Maxwell-Ampere law by replacing the volumetric susceptibility with $\chi^{e e}=\chi_{2 D}^{e e} \delta\left(z-z_{0}\right) \quad$ where $z_{0} \quad z_{0}$ is the location of metasurface in space and we have: $\tilde{N} \mathbf{H} . d \mathbf{l}=j \omega \dot{q}_{0} \iint\left(1+\chi_{2 D}^{e e} \delta\left(z-z_{0}\right)\right) d \mathbf{S}$. As a result, the relation between the tangential magnetic fields on both sides of the metasurface and the surface susceptibility is derived: $H_{x}^{+} l-H_{x}^{-} l=j \omega \varepsilon_{0}\left(h+\chi_{2 D}^{e e}\right) E_{y} l$. For the case of a homogenized layer, the induced susceptibility is uniformly distributed over the thickness (see Fig. S5), therefore we can replace the susceptibility with $\quad \chi^{e e}=\chi_{3 D}^{e e}, \quad \chi^{e e}=\chi_{3 D}^{e e} \prod\left(\frac{z-z_{0}}{t}\right), \quad$ which leads to $H_{x}^{+} l-H_{x}^{-} l=j \omega \varepsilon_{0}\left(h+\chi_{3 D}^{e e} t\right) E_{y} l$ after integration. Comparing results of Maxwell-Ampere law in both cases, it is clear that the relation between surface and volumetric electric susceptibility is as 
follows: $\chi_{3 D}^{e e}=\frac{\chi_{2 D}^{e e}}{t}$, and this derivation can simply be extended to surface and volumetric magnetic susceptibilities ${ }^{33}$. While these derivations are necessary to clarify the modeling, one can simply come to the final conclusion by considering the fact that the surface susceptibility should be uniformly distributed and averaged over the effective layer to form its volumetric counterpart, therefore simple average operation over the thickness $t$ is necessary. Finally, the tangential components of the electric permittivity and magnetic permeability of the nanocubic layer can be approximated from the reflection and transmission coefficients through the induced surface susceptibilities in the following way: $\varepsilon_{x x, y y}=\left(1+\frac{\chi_{x x, y y}^{e e}}{t}\right), \mu_{x x, y y}=\left(1+\frac{\chi_{x x, y y}^{m m}}{t}\right)^{33}$. Therefore the effective refractive index of the nanocube layer is $n_{t}=\sqrt{\varepsilon_{t} \mu_{t}}$, in which subscript $t$ denotes the tangential components. Fig. S6A and Fig. S6B illustrate the effective permittivity and permeability of the nanocube layer standing in free space, respectively. It is clear that the nanocube layer does not possess a magnetic response. Furthermore, negative values of permittivity at $600 \mathrm{~nm}$, describe the plasmonic resonance associated with nanocubes which helps with broadband absorption and resulting high field intensity of the designed structure. Fig. S6C shows the effective refractive index of the nanocube layer in which the effective refractive index is matched to unity, resulting in zero Fresnel reflection.
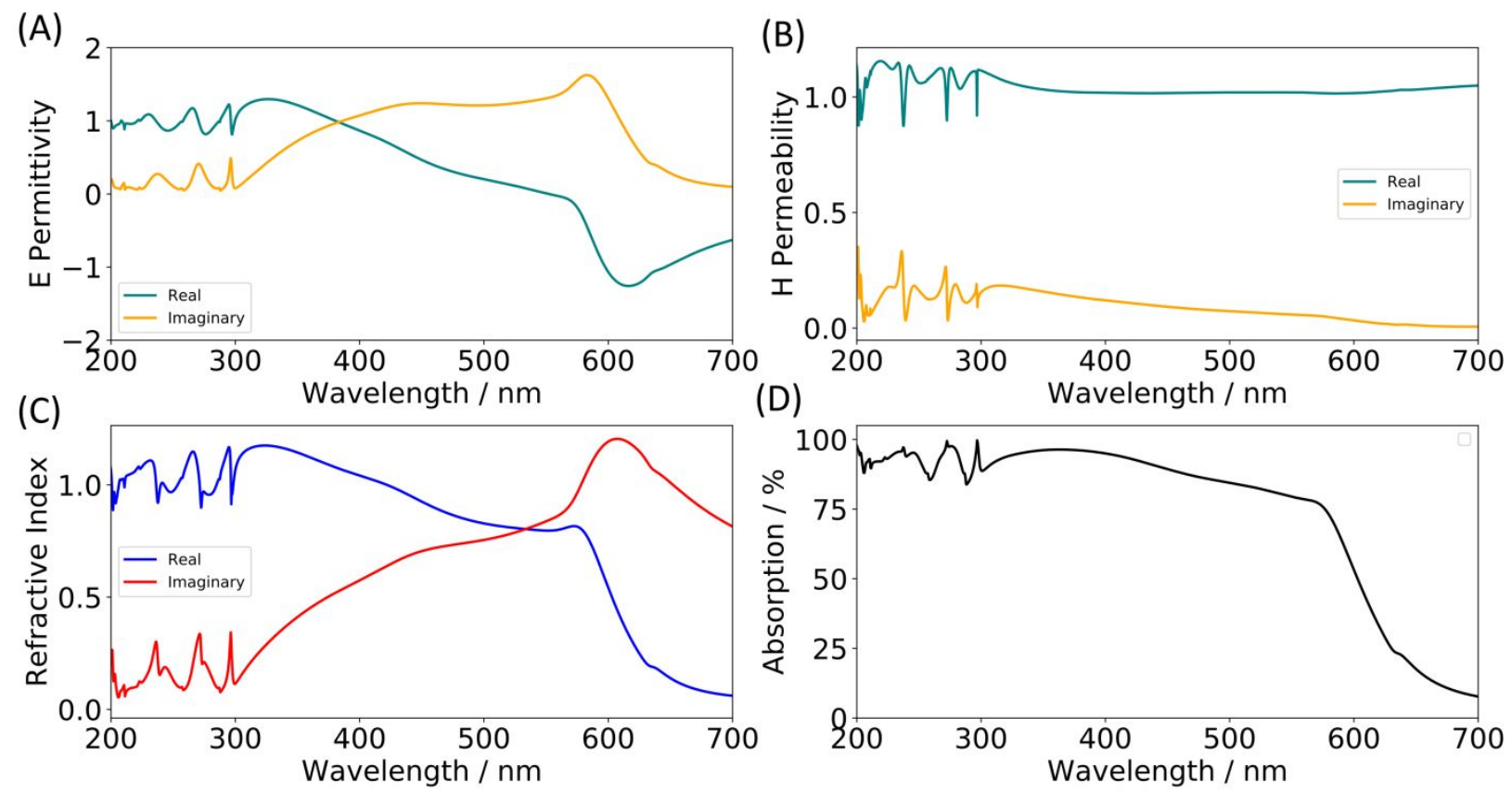

Fig. S6. Homogenized effective (A) electric permittivity, (B) magnetic permeability, and (C) refractive index of the nano-cube layer standing in free space without the underlying Au/Cu back mirror, and (D) the 
spectral absorption associated with the 3-layer metasurface model derived using the transfer matrix method.

With the derivation of the effective refractive index describing the copper nanocube layer, we can calculate the absorption of the entire metamaterial structure by virtue of the transfer matrix method (see Fig. S7D). Transfer matrix method relates the electric fields at the end and beginning of each layer through the following equations:

$$
\begin{aligned}
& \left(\begin{array}{l}
E(z+L) \\
H(z+L)
\end{array}\right)=M\left(\begin{array}{l}
E(z) \\
H(z)
\end{array}\right) \\
& M=M_{\text {Nanocube }} \cdot M_{A u} \cdot M_{C u}=\left(\begin{array}{cc}
A & B \\
C & D
\end{array}\right)
\end{aligned}
$$

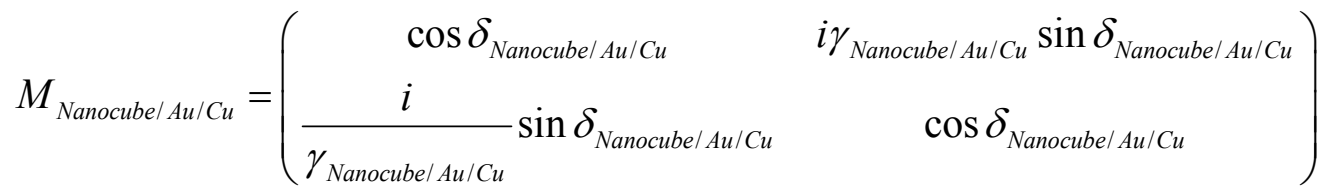

$$
\begin{aligned}
& \delta_{\text {Nanocube/ } / A u / C u}=k_{\text {Nanocube/Au/Cu}} L_{\text {Nanocube/Au/Cu }} \cos \theta_{\text {Nanocube/Au/Cu}} \\
& \gamma_{\text {Nanocube } / A u / C u}^{T E}=\frac{\eta_{\text {Nanocube } / A u / C u}}{\cos \theta_{\text {Nanocube } / A u / C u}} ; \gamma_{\text {Nanocube/Au/Cu}}^{T M}=\eta_{\text {Nanocube } / A u / C u} \cos \theta_{\text {Nanocube/Au/Cu}}
\end{aligned}
$$

where $\eta, \theta$ and $k$ are the intrinsic impedance, propagation angle, and propagation constant of the wave inside each layer, respectively. Subscripts Nanocube, $A u$, and $C u$ denote the effective parameters of the nanocube layer, gold layer, and the copper substrate, respectively. $L$ is the respective thickness of each layer. Total transmission and reflection are obtained using the following equations

$$
r=\frac{A+B / Z_{0}^{T E / T M}-C Z_{0}^{T E / T M}-D}{A+B / Z_{0}^{T E / T M}+C Z_{0}^{T E / T M}+D}
$$




$$
\begin{aligned}
& t=\frac{2(A D-B C)}{A+B / Z_{0}^{T E / T M}+C Z_{0}^{T E / T M}+D} \\
& Z_{0}^{T E}=\frac{Z_{0}}{\cos \theta_{i}} ; Z_{0}^{T M}=Z_{0} \cos \theta_{i}
\end{aligned}
$$

To compare our semi-analytical approach with the numerical simulations, we first derive the effective refractive index associated with the nanocube layer in free space (see Fig. S6C and Eqs. (S1-S5)) under normal incidence and use it to calculate the angular-spectral absorption associated with the entire metamaterial structure (see Fig. S7). Comparing the derived angular absorption coefficients under transverse electric and transverse magnetic illumination we see that the semi-analytical approach based on the generalized sheet boundary condition is a better technique for characterization of the metasurface absorber, when compared to the effective medium approach. However, there are still discrepancies between the results. This is due to the coupling effect between the back mirror and the nanocube layer which is not accounted for in the transfer matrix approach.

(A)

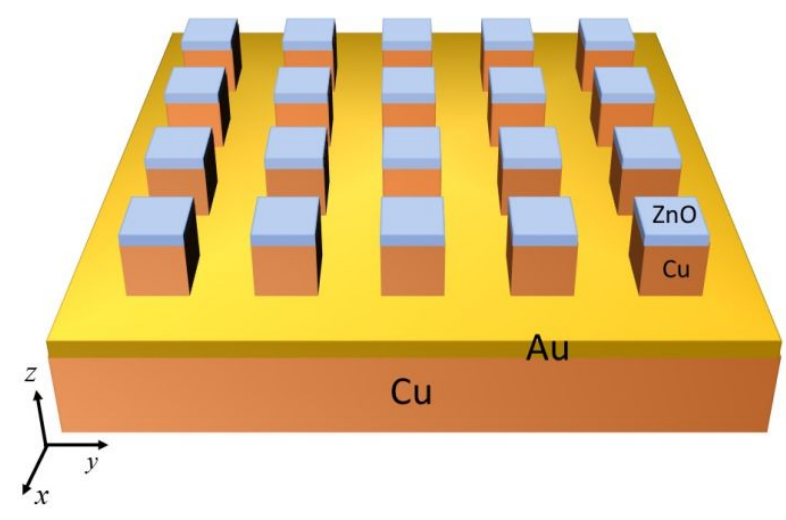

(B)

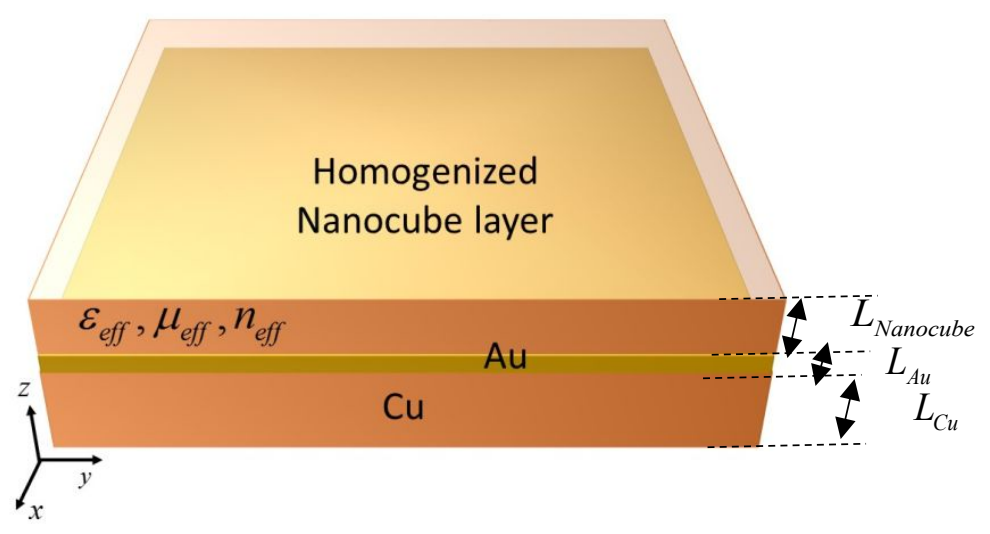

Fig. S7. (A) Metasurface near-perfect absorber and (B) 3-layer model based on the homogenized refractive index of the nano-cube layer.

Figure S8 shows that a uniformly coated stack of $\mathrm{ZnO} / \mathrm{Cu} / \mathrm{Au} / \mathrm{Cu}$ is significantly less absorptive, when compared to the designed metasurface. Furthermore, it is clear that the proposed impedance matching technique is capable of providing a design with wide angle wideband spectral absorption, which in turn is the basis of the demonstrated metacatalytic surface for conversion of $\mathrm{CO}_{2}$ into methanol under light. 


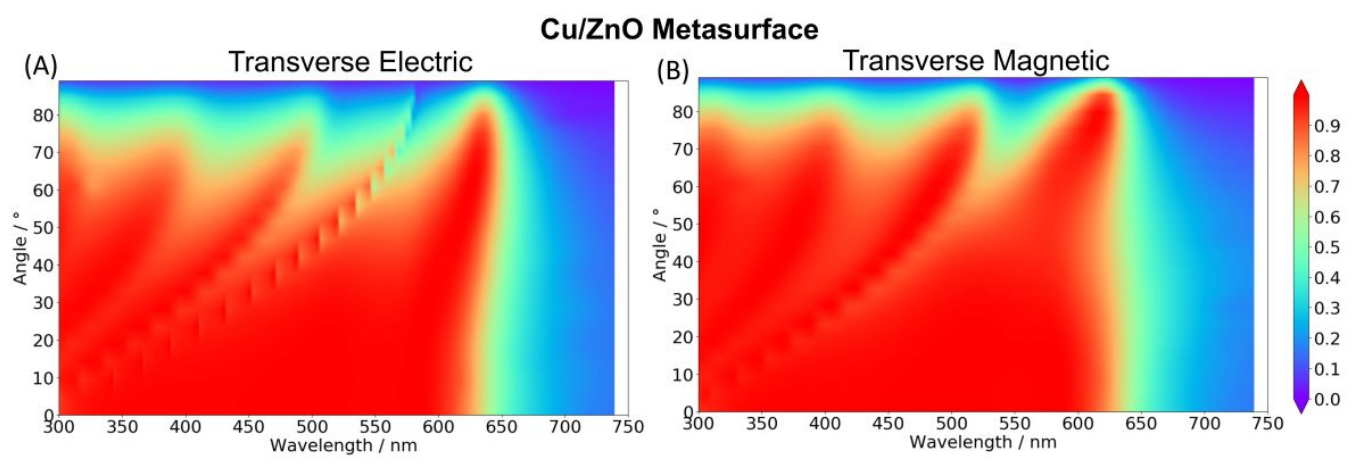

Effective Index Layer on $\mathrm{Au} / \mathrm{Cu}$
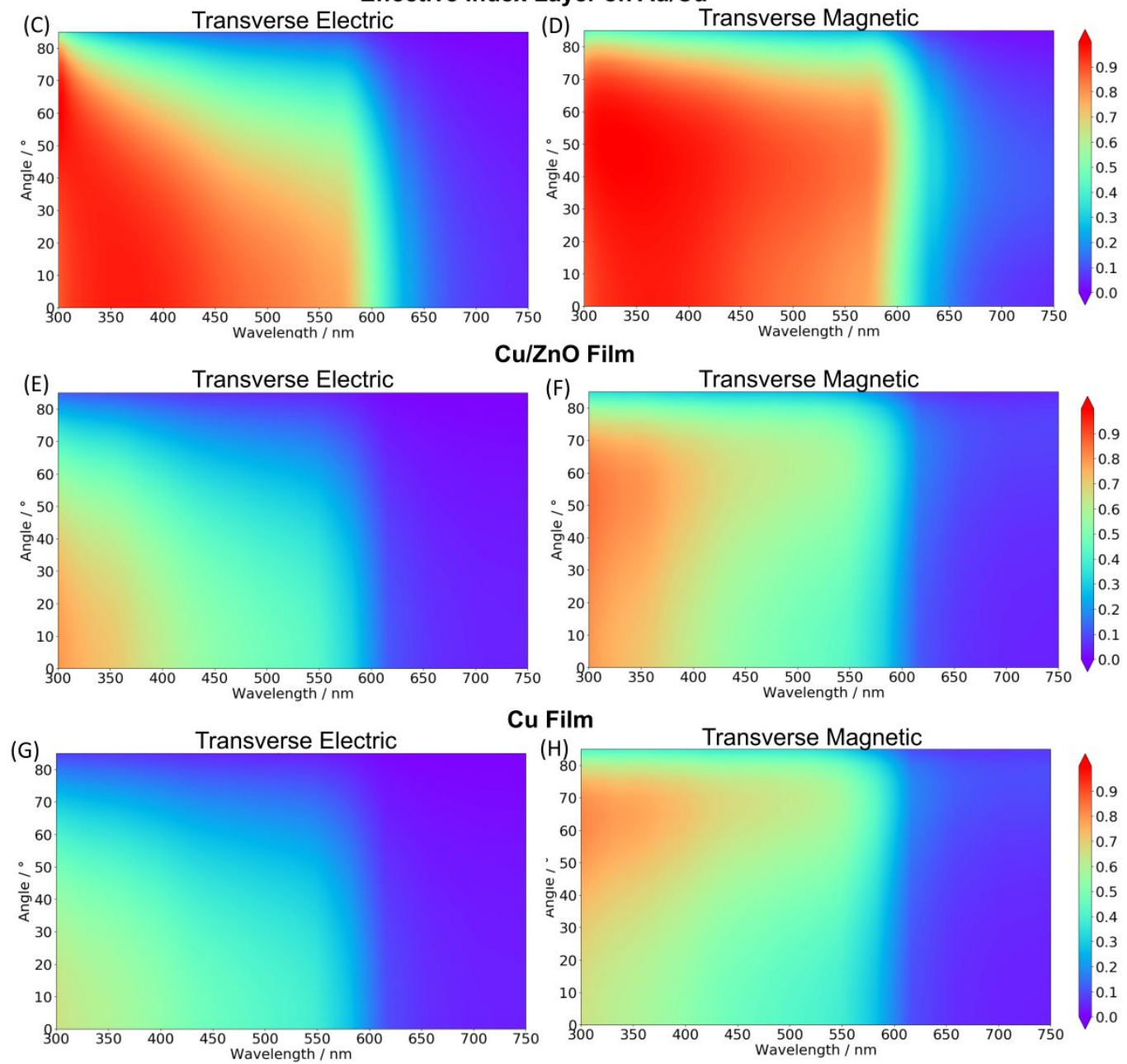

Fig. S8. Transverse Electric and Transverse Magnetic polarization angular-spectral absorption profiles of the (A, B) metasurface near perfect absorber, $(\mathbf{C}, \mathbf{D})$ the homogenized 3-layer model of the metasurface based on the green sheet boundary condition, (E, F) uniform multilayer $\mathrm{ZnO} / \mathrm{Cu} / \mathrm{Au} / \mathrm{Cu}$ stack, $(\mathbf{G}, \mathbf{H})$ and uniform multilayer $\mathrm{Cu} / \mathrm{Au} / \mathrm{Cu}$ stack. 


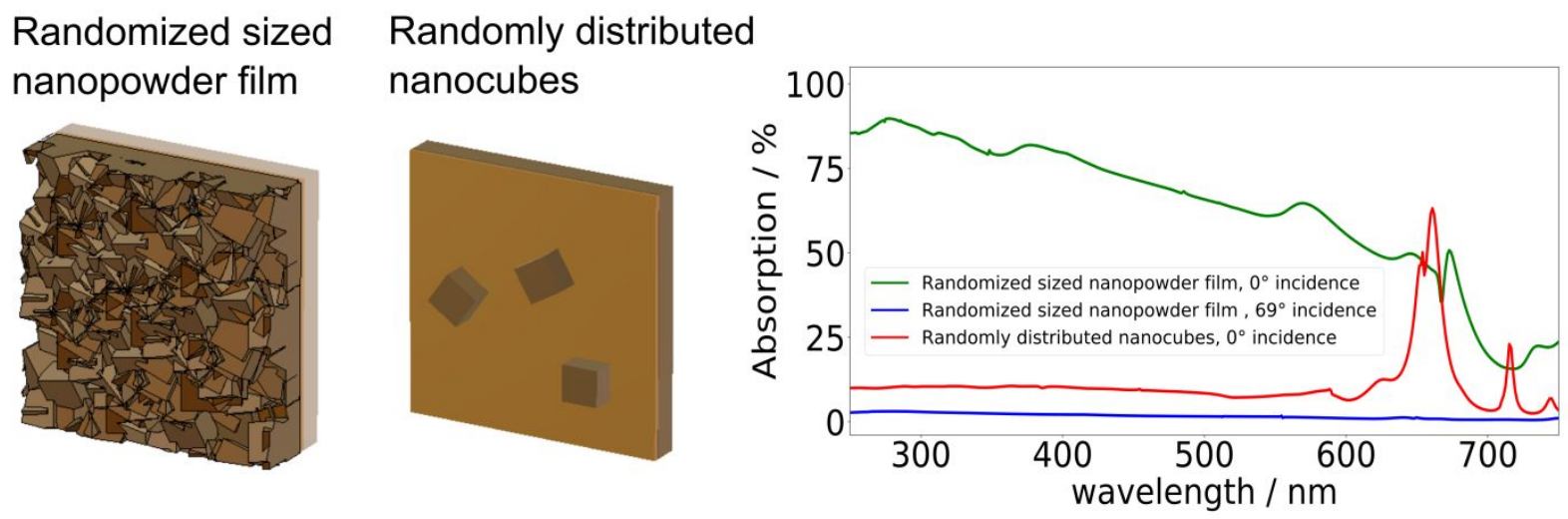

Fig. S9. Comparing in one plot the optical absorption of randomly sized copper packed nanopowder of nanocubes with a diameter of 30 to $140 \mathrm{~nm}$, and randomly distributed isolated copper nanocubes (150nm square with height of $100 \mathrm{~nm}$ ) with an areal density of $3 \mathrm{~mm}^{-2}$. Optical absorption spectra are shown for the nanopowder at normal incidence angle and glancing incidence angle (green and blue lines, respectively), and the distributed nanocubes at normal incidence angle (red line). The nanopowder film shows minimal absorption at a glancing angle of $69^{\circ}$ due to effective scattering of light by the randomly angled corners and edges of the nanocubes whereas the metamaterial showed high optical absorption similarly close to that of $0^{\circ}$ incidence. 